LoRenNa Viccentine COUTINHO MONTESCHOP2

Rosana Rosseto de Olivera ${ }^{3}$

Maria do Rosário Dias de Olivera Latorre ${ }^{4}$

Sandra Marisa Pelloso ${ }^{4}$

Thais Aidar de Frettas Mathias ${ }^{4}$

Artigo Original

Palavras-chave

Cesárea

Parto normal

Hospitais públicos

Hospitais privados

Declaração de nascimento Sistemas de informação

Keywords

Cesarean section Natural childbirth Hospitals, public Hospitals, private

Birth certificates Information systems

Gisele Ferreira Paris Universidade Estadual do Oeste do Paranó Rua Maringá, 1200 - Vila Nova

(EP: $85605-010$ Francisco Beltrĩo (PR), Brasi

Recebido

$28 / 05 / 2014$

Aceito com modificacōes

09/10/2014

\title{
Tendência temporal da via de parto de acordo com a fonte de financiamento
}

\author{
Time trend of the rates of cesarean and vaginal delivery \\ according to the source of financing
}

\section{Resumo}

OBJETIVO: Analisar a tendência temporal das taxas das vias de parto de acordo com a fonte de financiamento. MÉTODOS: Trata-se de um estudo ecológico de séries temporais de análise das taxas das vias de parto de acordo com a fonte de financiamento, no município de Maringá, Paraná, de 2002 a 2012 . Para coleta de dados foram utilizadas as informações disponíveis no Sistema de Informações sobre Nascidos Vivos e no Sistema de Informações Hospitalares do Sistema Único de Saúde (SUS). Para todas as taxas das vias de parto foram calculadas as médias móveis, para suavizar as oscilações aleatórias da série, feitos diagramas de dispersão entre os coeficientes e os anos de estudo, e a partir da relação funcional observada foram estimados modelos de regressão polinomial, com nível de significância de p<0,05. RESULTADOS: No decorrer dos 11 anos do estudo ocorreram 48.220 nascimentos. Desses, 77, 1 \% foram parto cesáreo e apenas 22,9\% parto vaginal. Os partos financiados pelo SUS totalizaram 22.366 procedimentos e desses, $54,6 \%$ foram cesáreas. A análise da tendência foi significativa para todos os modelos de regressão, evidenciando tendência ascendente para parto cesáreo e decrescente para parto vaginal nos dois tipos de financiamento. Observa-se que as taxas de cesárea não SUS foram sempre superiores a 90,0\% e mais frequentes do que as cesáreas SUS, mesmo com o aumento de 36,0\% dessas ao longo do período estudado. CONCLUSÃO: Pela análise de tendência, as cesáreas continuarão aumentando nos dois financiamentos de saúde se não forem implantadas novas ações e estratégias de redução envolvendo as características socioculturais, demográficas e obstétrica das mulheres, a formação e a atuação profissional na obstetrícia e a estrutura adequada dos serviços de saúde para atendimento ao parto vaginal.

\section{Abstract}

PURPOSE: To analyze the time trend of the rates of cesarean and vaginal delivery according to the source of financing. METHODS: This was an ecological study of the time series analysis of cesarean and vaginal delivery rates according to the financing source, carried out in Maringá, Paraná State, Brazil, from 2002 to 2012 . Information available at the System of Information on Live Births and at the System of Hospital Information of the Brazilian Unified Health System (SUS) was used for data collection. Moving averages were calculated for all mode of delivery rates in order to smooth random fluctuations in the series, dispersion diagrams were designed between the coefficients and years of the study, and polynomial regression models were estimated from the functional relation observed, with the level of significance set at $p<0.05$. RESULTS: Throughout the 11 years of the study there were 48,210 births, $77.1 \%$ by cesarean delivery and only $22.9 \%$ by vaginal delivery. A total of 22,366 procedures were financed by SUS, $54.6 \%$ of them being cesareans. Trend analysis was significant for all the regression models, demonstrating an ascending trend for cesarean delivery and a descending trend for vaginal delivery for both types of financing. The non-SUS cesarean rates always exceeded $90.0 \%$ and were more frequent than the SUS cesarean rates, even with a $36.0 \%$ increase of the latter during the study period. CONCLUSION: Based on trend analysis, cesarean deliveries will continue to increase in both health financing sources unless new actions and strategies of reduction are implemented, involving the sociocultural, demographic and obstetric characteristics of women, the training and activity of professionals in the area of obstetrics and an adequate structure of health services for providing vaginal delivery.

\section{Programa de Pós-Graduação em Enfermagem da Universidade Estadual de Maringá - UEM - Maringá (PR), Brasil.}

'Universidade Estadual do Oeste do Paraná - Unioeste - Francisco Beltrão (PR), Brasil.

${ }^{2}$ Hospital Universitário de Maringá, Universidade Estadual de Maringá - UEM - Maringá (PR), Brasil.

3Programa de Pós-Graduação em Enfermagem, Universidade Estadual de Maringá - UEM - Maringá (PR), Brasil.

4Programa de Pós-Graduação em Epidemiologia, Universidade de São Paulo - USP - São Paulo (SP), Brasil.

Conflito de interesses: não há 


\section{Introdução}

Estudos recentes ${ }^{1,2}$ confirmam a taxa de cesárea medicamente justificada de até $15 \%$ conforme a preconização da Organização Mundial de Saúde (OMS) desde 1985. No Brasil, apesar do aprimoramento da assistência ao longo do tempo, observa-se elevadas e crescentes as taxas de cesárea: 38\% em 2000, 43\% em 2005, 52\% em 2010 e $54 \%$ em $2011^{3}$.

Apesar do benefício da cesárea de prevenir agravos e salvar vidas, vários estudos ${ }^{4-10}$ vêm abordando associação do seu uso indiscriminado e a morbimortalidade materna e infantil. Estudos atribuem também efeitos em curto e longo prazo da cesárea na saúde das mulheres e crianças, como maior proporção de trabalho de parto prematuro e infecção puerperal ${ }^{11}$, maior risco no segundo parto após cesárea planejada para hemorragia, ruptura uterina e óbito fetal ${ }^{5}$, incidência de prematuridade e baixo peso ao nascer ${ }^{6}$, e até possibilidade de associação com o sobrepeso na infância ${ }^{7}$, adolescência e na idade adulta jovem ${ }^{8}$.

$\mathrm{Na}$ última década, no estado do Paraná, dentre os óbitos maternos mais de $65 \%$ das mulheres haviam sido submetidas ao parto cesáreo, o que significou um risco de 70 óbitos maternos por 100 mil nascidos vivos e um risco relativo de morte por cesárea em relação ao parto normal 1,9 vezes maior, independente da causa do óbito materno $^{3,9}$. Em relação à mortalidade infantil, entre 2005 e 2006, na 15ª Regional de Saúde do Estado do Paraná, a redução do óbito infantil foi de $83 \%$ em relação ao parto normal e de $70 \%$ na cesárea ${ }^{10}$.

Para o governo brasileiro, a elevada taxa de cesárea no país representa um grande desafio, considerando os riscos desnecessários tanto para a mãe quanto para a criança, além da sua associação com a mortalidade materna e os custos adicionais para o sistema de saúde; principalmente quando a redução da mortalidade materna está associada à atenção qualificada ao parto, à assistência obstétrica de emergência e à impossibilidade do Brasil em atingir a meta do milênio de redução de $75 \%$ da mortalidade materna até 2015.

Dentre as recomendações para prevenção segura da primeira cesariana, o Conselho Americano de Ginecologia e Obstetrícia menciona a necessidade de rever a definição de distócia de trabalho parto, pois dados recentes mostram que o trabalho de parto contemporâneo avança a um tempo mais lento do que o que foi tradicionalmente ensinado. Acresenta a necessidade de aumentar o acesso das mulheres a intervenções não médicas durante o trabalho de parto e a intervenção de versão cefálica externa na apresentação pélvica ${ }^{11}$.

Considerando a necessidade de estudos sobre a proporção de cesáreas ${ }^{12}$, de sua associação com a morbimortalidade materna e neonatal, e que o município de
Maringá, pela concentração de serviços especializados e instituições de ensino de saúde, representa uma referência na gestão, apoio e planejamento das ações em saúde para a macrorregião noroeste do estado do Paraná, foi proposto o presente estudo com o objetivo de analisar a tendência temporal das taxas das vias de parto de acordo com a fonte de financiamento em uma cidade da região Sul do Brasil.

\section{Métodos}

Trata-se de um estudo ecológico de séries temporais de análise das taxas das vias de parto de acordo com a fonte de financiamento, de residentes no município de Maringá, Paraná, de 2002 a 2012. Para a coleta de dados foram utilizadas as informações disponíveis no site do Departamento de Informática do Sistema Único de Saúde (SUS) (DATASUS) ${ }^{3}$, no Sistema de Informações sobre Nascidos Vivos (SINASC) de abrangência e obrigatoriedade nos serviços públicos e privados e no Sistema de Informações Hospitalares do Sistema Único de Saúde (SIH-SUS) de todas as internações realizadas em território nacional financiadas pelo SUS.

Apesar do SINASC abranger todos os nascimentos no Brasil, não existe nesse sistema um campo referente à informação sobre a fonte de financiamento do parto. No SINASC foi selecionada ocorrência do parto no "município de Maringá-PR" e foram incluídos todos os tipos de "parto vaginal”, "cesáreo", "fórceps, outros" e "ignorados".

Para coleta de dados no SIH-SUS foi utilizada a ferramenta do Ministério da Saúde "Tabnet" procedimentos hospitalares por local de residência, para os anos de 2002 a 2007 na categoria procedimento obstétrico, "parto normal" e "parto cesáreo". Devido às mudanças no SIH-SUS a partir de 2008, foram selecionados os procedimentos hospitalares por local de residência "parto cesáreo em gestação de alto risco", "parto cesáreo", "parto cesáreo com laqueadura tubária", "parto normal" e "parto normal em gestação de alto risco". Não foi selecionado "parto normal em centro de parto normal" devido à inexistência no município e nenhum caso registrado.

Foi considerado "parto SUS" quando os custos foram financiados pelo SUS, e "parto não SUS" quando pagos diretamente pela cliente ou indiretamente através de convênios ou sistema de seguro saúde. Para o cálculo da taxa total dos partos SUS (TTPSUS) foi considerado, para cada ano, a razão entre o número total de partos no SIH pelo número total de nascimentos constante no SINASC multiplicado por cem, como segue: TTPSUS $=\mathrm{n}$ total de partos no SIHx100/n total de nascimentos no SINASC.

Para o cálculo da taxa total dos partos não SUS (TTPNSUS), foi considerada, para cada ano, a diferença entre o número total de nascimentos constante no SINASC pelo número total de partos no SIH, pela razão do número total de nascimentos constante no SINASC multiplicado 
por cem, como segue: TTPNSUS $=\mathrm{n}$ total de nascimentos no SINASC-n total de partos do SIHx100/n total de nascimentos no SINASC.

Os métodos de cálculo demonstrados anteriormente foram os denominadores para o cálculo das taxas de parto vaginal e parto cesáreo segundo o tipo de financiamento, conforme calculados a seguir. Para as taxas de parto vaginal e parto cesáreo SUS (TPVSUS/TPCSUS) foram calculadas, para cada ano, a razão entre o número de nascimentos segundo cada tipo de parto constantes no banco do SIH-SUS pelo número total de nascimentos SUS multiplicado por cem, como segue: TPVSUS $=\mathrm{n}$ parto vaginal SUS (no SIH-SUS) $\mathrm{x} 100 / \mathrm{n}$ total de nascimentos SUS e TPCSUS $=\mathrm{n}$ cesárea SUS (no SIH-SUS)x100/n total de nascimentos SUS.

Para as taxas de parto vaginal e parto cesáreo não SUS (TPVNSUS/TPCNSUS), foi calculada, para cada ano, a diferença entre o número de nascimentos de cada tipo de parto constantes no banco do SINASC pelo número de cada tipo de parto constantes no banco do SIH-SUS, pela razão do número total de nascimentos não financiados pelo SUS multiplicado por cem, como segue: TPVNSUS $=\mathrm{n}$ parto vaginal no SINASC-n parto vaginal no SIH-SUSx100/n total de nascimentos não SUS e TPCNSUS =n parto cesáreo no SINASC-n parto cesáreo no SIH-SUSx100/n total de nascimentos não SUS.

Para o total das taxas de parto vaginal (TTPV) e parto cesáreo (TTPC), foram calculadas, para cada ano, a soma individual de cada tipo de parto, pela razão do número total de nascimentos multiplicado por 100, como segue: $T T P V=n$ parto vaginal no SUS $+n$ parto vaginal no não $S U S x 100 / n$ total de nascimentos e TTPC $=\mathrm{n}$ parto cesáreo no $\mathrm{SUS}+\mathrm{n}$ parto cesáreo no não SUSx100/n total de nascimentos.

As taxas de parto cesáreo e parto vaginal foram consideradas como variável dependente $(\mathrm{Y})$, e os anos do calendário de estudo como variável independente (X). Para todas as taxas das vias de parto foram calculadas as médias móveis de três pontos para alisamento dos dados. $\mathrm{O}$ alisamento consiste em uma técnica não paramétrica e tem a propriedade de suavizar as oscilações aleatórias da série. Inicialmente, foram feitos diagramas de dispersão entre os coeficientes e os anos de estudo para visualizar a função que poderia estar expressando a relação entre eles. A partir da relação funcional observada foram estimados modelos de regressão polinomial que, além do seu poder estatístico, apresentam fácil elaboração e interpretação.

No processo de modelagem testou-se o modelo de regressão linear simples $(Y=\beta 0+\beta 1 \mathrm{X})$ e em seguida os modelos de segundo grau $\left(Y=\beta 0+\beta 1 X+\beta 2 X^{2}\right)$ e terceiro grau $\left(Y=\beta 0+\beta 1 X+\beta 2 X^{2}+\beta 3 X^{3}\right)$. Quando dois modelos foram semelhantes do ponto de vista estatístico optou-se pelo modelo mais simples, ou seja, o de menor ordem observando o princípio da parcimônia. Considerou-se tendência significativa aquela cujo modelo estimado obteve $p<0,05$. Nesses modelos, $\beta 0$ é o coeficiente anual médio, $\beta 1$ é o coeficiente de efeito linear (velocidade) e $\beta 2$ o coeficiente de efeito quadrático (aceleração).

A transformação da variável ano em ano-centralizado é necessária nos modelos de regressão polinomial observando os termos da equação que frequentemente são altamente correlacionados e expressam a variável independente como um desvio de sua média, reduzindo substancialmente a autocorrelação entre eles. $\mathrm{O}$ ano médio do período foi 2007. Como medida de precisão do modelo utilizou-se o coeficiente de determinação $\left(\mathrm{r}^{2}\right)$, considerando o modelo mais ajustado quando o $\mathrm{r}^{2}$ está mais próximo de 1 . Os cálculos das taxas nas séries históricas foram elaborados em planilhas do Excel (versão 7.0 para Windows 95) e as análises de tendências realizadas no Statistical Package for Social Sciences (SPSS), versão 20.0.

O estudo seguiu as normas de pesquisa envolvendo seres humanos conforme Resolução $n^{\circ} 196 / 96$, com dispensa da assinatura do Termo de Consentimento Livre e Informado por utilizar fontes secundárias. Foi aprovado pelo Comitê de Ética em Pesquisa da Universidade Estadual de Maringá sob o parecer n ${ }^{\circ} 410.866 / 2013$.

\section{Resultados}

No decorrer dos 11 anos do estudo, segundo o SINASC, ocorreram 48.220 nascimentos no município de Maringá, Paraná. Desses, $77,1 \%$ foram por parto cesáreo e apenas $22,9 \%$ por parto vaginal. Em toda a série histórica, apenas dois partos no ano de 2002 foram classificados como ignorados, mesmo com a exclusão desse campo em 2001. Os partos financiados pelo SUS, conforme o SIH-SUS, totalizaram 22.366 procedimentos e desses, 54,6\% foram cesáreas.

Quanto às frequências absolutas e relativas dos tipos de parto segundo as fontes de financiamento, observa-se que as taxas de parto cesáreo não SUS foram sempre superiores a 90,0\% e mais frequentes do que os partos cesáreos financiados pelo SUS. Essa diferença se manteve mesmo com o aumento de 36,0\% na taxa de cesáreas do SUS, de 41,9 para $65,3 \%$ ao longo do período estudado. Para o parto vaginal, as taxas diminuíram tanto no SUS, de 58,1 para $34,7 \%$, quanto no não SUS, de 6,3 para $2,2 \%$. Embora as proporções de partos vaginais e cesáreos no SUS modificarem ao longo do período, a partir do ano de 2005 mantiveram-se em percentuais inferiores em todos os anos (Tabela 1).

$\mathrm{Na}$ análise da tendência observou-se que esta foi significativa para todos os modelos de regressão, evidenciando tendência ascendente para parto cesáreo SUS de 1,9\% ao ano e de $0,2 \%$ ao ano para parto cesáreo não SUS, que já era alto no início do período, e tendência decrescente igual para parto vaginal SUS e não SUS de $0,3 \%$ ao ano (Figura 1 ). 
Tabela 1. Distribuicão dos partos ocorridos em Maringá, Paraná, segundo o tipo de parto, ano e fonte de financiamento

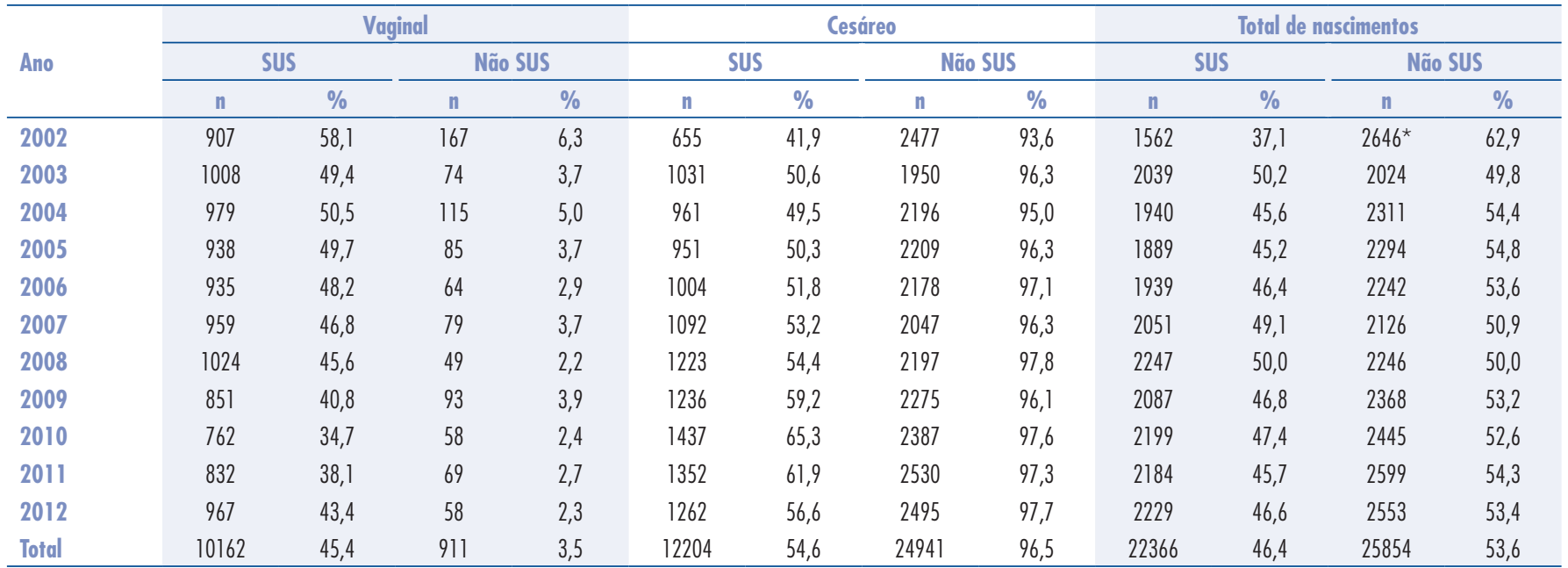

SUS: Sistema Único de Saúde; *dois tipos de parto ignorados.

Fonte: Ministério da Saúde. Departamento de Informática do SUS (DATASUS) ${ }^{3}$.

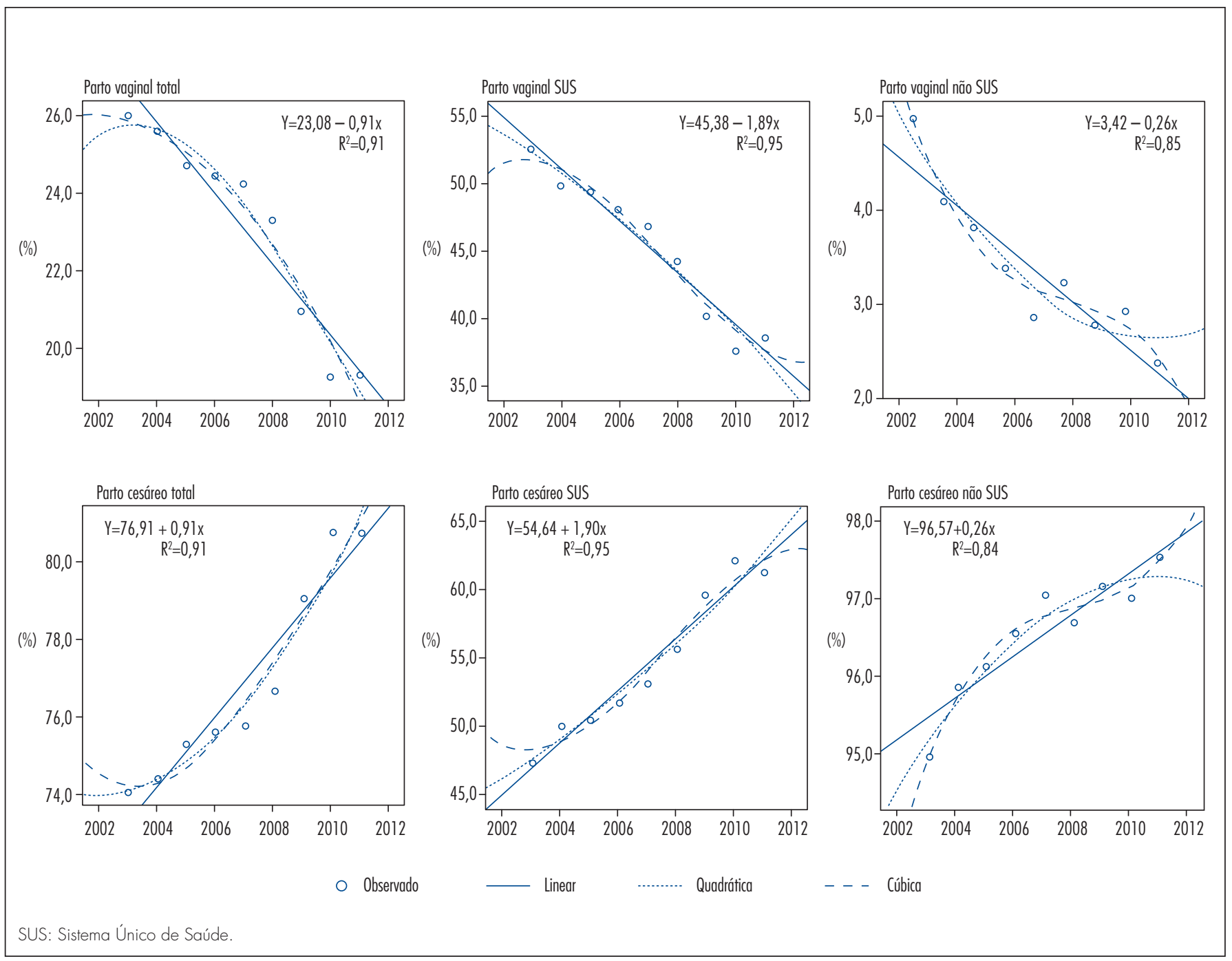

Figura 1. Diagramas de dispersão das taxas de parto vaginal e cesáreo segundo financiamento do parto 


\section{Discussão}

O presente estudo apresentou a trajetória dos tipos de parto financiados ou não pelo SUS para o município de Maringá, Paraná, de 2002 a 2012 e possibilitou inferir provável realidade de uma série futura de acordo com a observação no período, de cada quatro crianças nascidas, três foram por parto cesáreo. A taxa de parto cesáreo de $78,6 \%$ em 2012 representa 5,2 vezes mais do que o recomendado para as mulheres de risco habitual (15\%) e 3,1 vezes mais para as mulheres de alto risco $(25 \%)$ conforme a $\mathrm{OMS}^{13}$. A tendência ascendente do parto cesáreo em quase todas as regiões do Brasil é considerada uma prática indiscriminada e epidêmica ${ }^{14}$.

$\mathrm{Na}$ comparação entre as taxas de parto cesáreo em diferentes países do mundo, o Brasil ocupa a liderança numérica, com taxas superiores tanto em relação aos países considerados como prestadores de assistência menos intervencionista ${ }^{15}$ (Holanda e República Tcheca com 14\%) quanto aos países com assistência mais intervencionista, como Canadá com 23\% e Estados Unidos com $25 \%$. Com as altas taxas de parto cesáreo desde o início do período do estudo, o aumento de no máximo $7 \%$ na taxa total de cesáreas na série histórica do município de Maringá apresentou-se inferior ao aumento de $16 \%$ no Brasil ${ }^{14}$, e superior a outras localidades do mundo. A proporção de aumento variou menos de $1 \%$ para Finlândia, Coréia e Bélgica, 2,4\% para Holanda, 3,1\% Canadá e 4,4\% para os Estados Unidos ${ }^{15}$.

Quando comparamos a taxa de parto cesáreo no Brasil em relação a outros países que têm as menores taxas de cesariana, por exemplo, Inglaterra e Holanda, não podemos esquecer que esses países dispõem de diferente regulação das práticas médicas e, sobretudo, modelos de atenção à saúde materna nos quais a gestante de risco habitual é primariamente atendida por parteiras profissionais de nível universitário com midwifery programmes ${ }^{16}$.

Quanto às frequências de 65,3\% de parto cesáreo no SUS e de 97,8\% no serviço não SUS deste estudo, são muito superiores principalmente quando comparadas às taxas de cesárea em outra realidade, do extremo Sul do Brasil, de $36 \%$ no SUS e de $81 \%$ no privado ${ }^{12}$, como também na série histórica do Brasil, ${ }^{17} \mathrm{com}$ a taxa de $27 \%$ de cesárea no SUS e de $80 \%$ no serviço suplementar. Há também diferenças em relação à Argentina, com a taxas de cesárea, respectivamente nos serviços públicos e privados, de 24 e $52 \%$.

Os padrões das taxas de partos cesáreos descritos neste estudo são compatíveis com a hipótese proposta por Barros et al. ${ }^{12}$ de que as cesáreas são feitas, em grande parte, para atender à conveniência das agendas dos médicos, levando em consideração que a maior parte das cesáreas ocorreram no período diurno ${ }^{4}$. Embora estudos iniciem abordando frequência das cesáreas a pedido da mulher ${ }^{18}$, as decisões pelo tipo de parto devem considerar as preferências das gestantes, desde que elas tenham condições de escolher de forma isenta o que melhor lhes convém ${ }^{19}$. No Brasil, em um centro de parto normal intra-hospitalar no qual a assistência ao parto está a cargo de enfermeiras obstétricas, em que é possível supor que o local foi escolhido pelas gestantes desde que não apresentassem riscos, foi encontrada a prevalência de cesáreas de $14,9 \%$, dentro dos limites propostos pela $\mathrm{OMS}^{20}$. Para o município de estudo, espera-se que a cobrança pelos convênios de saúde para que o mesmo médico acompanhe o pré-natal e parto seja uma medida para redução da cesárea, pois assim não será possível estabelecer um agendamento para a cesárea.

As mulheres justificam a escolha pelo parto normal pela recuperação mais rápida e para a cesárea pelo medo de sentir dor. Contudo, por trás dessas escolhas há falta de esclarecimento para as mulheres sobre os tipos de parto e a possibilidade de anestesia e analgesia em ambos os $\operatorname{casos}^{21}$, e quanto às complicações pós-cirúrgicas da cesariana, principalmente nas mulheres em condições sociais e de saúde menos privilegiadas ${ }^{22}$.

Estudos apontaram que um dos itens responsáveis pela maior redução da mortalidade infantil seria o nascimento por parto normal ${ }^{10,21}$, o que demonstra a necessidade de rever os currículos da área obstétrica e o preparo para os atendimentos de parto normal ${ }^{23}$. Muito há que se pensar nos mecanismos institucionais e de organização das redes de saúde para o resgate da cultura voltada ao parto natural ${ }^{24}$ e do sistema de educação médica que valoriza a medicalização da saúde colaborando para a falta de preparo de médicos e profissionais da saúde na condução ao parto normal de forma mais fisiológica ${ }^{25}$.

Embora tendência decrescente do parto vaginal, a associação entre o tipo de parto e a qualidade da atenção segundo indicadores preconizados pelas OMS demonstrou que as mulheres em Maringá, Paraná, submetidas ao parto vaginal receberam atenção mais qualificada quando comparadas às que foram à cesárea ${ }^{26}$. Já em outra realidade brasileira, os resultados indicaram má qualidade da assistência ao parto vaginal, frequência não adequada de intervenções benéficas e alta ocorrência de algumas intervenções consideradas prejudiciais na assistência ao parto vaginal ${ }^{27}$.

A literatura indica um paradoxo perinatal ${ }^{28,29}$ devido à super utilização de tecnologias e os maiores gastos na atenção perinatal, demonstrado pelas altas taxas de cesáreas e maior número internação nas Unidades de Terapia Neonatal, com limitada melhora dos indicadores maternos e perinatais ${ }^{28}$. Os resultados apontam Maringá como um município com uso predominante de tecnologias, pelo aumento do número de partos cesáreos independente do tipo de financiamento do parto, disponibilizando a 
tecnologia também para as mulheres que são atendidas pelo sistema público de saúde no pré-natal e parto.

Dentre as limitações do presente estudo pode-se citar a utilização de dados em banco de dados secundário e a não realização de linkage entre os bancos de dados. Contudo, apesar dos estudos de séries temporais não propiciarem mensuração a nível individual, foi proposto o presente estudo devido à implantação das ações pelo Ministério da Saúde em 2000 para diminuição das cesáreas por meio do lançamento do Programa de Humanização no Pré-natal e Nascimento (PHPN), estruturação das centrais de regulação de leitos obstétricos e neonatais, pacto nacional pela redução das taxas de cesáreas e ações para alcance das metas dos objetivos do milênio de reduzir a mortalidade infantil e melhorar a saúde materna ${ }^{4}$.

Conclui-se que as altas taxas de cesárea e a tendência ascendente e prospectiva são o cenário atual de Maringá e podem ser a realidade de outros municípios de médio porte com características semelhantes no Brasil. Apesar das várias iniciativas do setor público com políticas e recomendações do SUS de incentivo ao parto vaginal e diminuição das taxas de cesáreas, a perspectiva de redução ainda não é vislumbrada. São necessárias políticas públicas mais efetivas para evitar a realização desnecessária desse procedimento cirúrgico. O SUS, que anteriormente equilibrava os índices de cesárea, agora atua como incremento na manutenção das altas prevalências.

Pela análise de tendência realizada neste estudo os partos cesáreos continuarão aumentando nos dois sistemas de saúde se não forem implantadas novas ações e estratégias de redução envolvendo as características socioculturais, demográficas e obstétricas das mulheres, a formação e atuação profissional na obstetrícia e a estrutura adequada dos serviços de saúde para atendimento ao parto vaginal. Os resultados deste estudo retratam o aumento do parto cesáreo ao longo do tempo, mas para explicação do fenômeno na saúde da população são necessários novos estudos.
1. Ye J, Betrán AP, Guerrero Vela M, Souza JP, Zhang J. Searching for the optimal rate of medically necessary cesarean delivery. Birth. 2014;41(3):237-44.

2. Gibbons L, Belizan JM, Lauer JA, Betran AP, Merialdi M, Althabe $F$. Inequities in the use of cesarean section deliveries in the world. Am J Obstet Gynecol. 2012;206(4):331.e1-19.

3. Brasil. Ministério da Saúde. DATASUS [Internet]. Informações de Saúde. Estatísticas vitais. Nascidos vivos desde 2000. Brasília (DF): Ministério da Saúde; 2014. [citado 2013 Dez 12]. Disponível em: <http://tabnet.datasus.gov.br/cgi/tabcgi.exe?sinasc/cnv/ nvpr.def>

4. Brasil. Ministério da Saúde. Secretaria de Vigilância em Saúde. Departamento de Análise de Situação de Saúde [Internet]. Saúde Brasil 2011: uma análise da situação de saúde e a vigilância da saúde da mulher. Capítulo 16: as cesarianas no Brasil: situação no ano de 2010, tendências e perspectivas. Brasília (DF): Editora do Ministério da Saúde; 2012. p. 371-98 [citado 2013 Maio 12]. Disponível em: <http://repositorio.unb.br/bitstream/10482/12834/1/ CAPITULO_CesarianasNoBrasil.pdf>

5. Kok N, Ruiter L, Hof M, Ravelli A, Mol BW, Pajkrt E, et al. Risk of maternal and neonatal complications in subsequent pregnancy after planned caesarean section in a first birth, compared with emergency caesarean section: a nationwide comparative cohort study. BJOG. 2014;121(2):216-23.

6. Barros FC, Victora CG, Barros AJ, Santos IS, Albernaz E, Matijasevich $A$, et al. The challenge of reducing neonatal mortality in middle-income countries: findings from three Brazilian birth cohorts in 1982, 1993, and 2004. Lancet. 2005;365(9462):847-54

7. Li H, Ye R, Pei L, Ren A, Zheng X, Liu J. Caesarean delivery, caesarean delivery on maternal request and childhood overweight: a Chinese birth cohort study of 181380 children. Pediatr Obes. 2014;9(1):10-6.
8. Barros FC, Matijasevich A, Hallal PC, Horta BL, Barros AN, Menezes $A B$, et al. Cesarean section and risk of obesity in childhood, adolescence, and early adulthood: evidence from 3 Brazilian birth cohorts. Am J Clin Nutr. 2012;95(2):465-70.

9. Brasil. Ministério da Saúde. DATASUS. Informações de Saúde. Óbitos de mulheres em idade fértil e óbitos maternos - Paraná [Internet]. Brasília (DF): Ministério da Saúde; 2014. [citado 2014 Out 21]. Disponível em: <http://tabnet.datasus.gov.br/cgi/ deftohtm.exe?sim/cnv/mat10pr.def>

10. Mathias TAF, Assunção AN, Silva GF. [Infant deaths investigated by the Prevention Committee of Infant Mortality in region of Paraná state]. Rev Esc Enferm USP. 2008;42(3):445-53. Portuguese.

11. American College of Obstetricians and Gynecologists, Society for Maternal-Fetal Medicine. Obstetric care consensus no. 1: safe prevention of the primary cesarean delivery. Obstet Gynecol. 2014;123(3):693-711.

12. Barros AJ, Santos IS, Matijasevich A, Domingues MR, Silveira M, Barros FC, et al. Patterns of deliveries in a Brazilian birth cohort: almost universal cesarean sections for the better-off. Rev Saúde Pública. $2011 ; 45(4): 635-43$.

13. World Health Organization (WHO). Appropriate technology for birth. Lancet. 1985;2(8452):436-7.

14. Höfelman DA. Tendência temporal de partos cesáreos no Brasil e suas regiões: 1994 a 2009. Epidemiol Serv Saúde. 2012;21 (4):561-8.

15. Vadeboncoeur $\mathrm{H}$. Une autre césarienne ou un avac? S`informer pour mieux décider. Montréal: FIDES; 2012.

16. Narchi NZ, Silva LCFP, Gualda DMR. Contexto, desafios e perspectivas na formação de obstetrizes no Brasil. Saúde Soc. 2012;21 (2):510-9.

17. Brasil. Agência Nacional de Saúde Suplementar. Brasil tem uma das maiores taxas de cesariana na saúde suplementar. Brasília (DF): ANS; 2006 [citado 2013 Nov 27]. Disponível em: <http://www. ans.gov.br/portal/site/home2/destaque_22585_2.asp> 
18. American College of Obstetricians and Gynecologists. ACOG Committee Opinion no. 559: cesarean delivery on maternal request. Obstet Gynecol. 2013:121(4):904-7.

19. Patah LEM, Malik AM. Models of childbirth care and cesarean rates in different countries. Rev Saúde Pública. $2011 ; 45(1): 185-94$.

20. Osava RH, Silva FM, Tuesta EF, Oliveira SM, Amaral MC. Cesarean sections in a birth center. Rev Saúde Pública. $2011 ; 45(6): 1036-43$.

21. Cardoso PO, Alberti LR, Petroianu A. [Nneonatal and maternal morbidity related to the type of delivery]. Ciênc Saúde Coletiva. 2010;15(2):42735. Portuguese.

22. Freitas PF, Savi EP. [Social inequalities in post-cesarean complication rates: a hierarchical analysis]. Cad Saúde Pública. $2011 ; 27(10): 2009$ 20. Portuguese.

23. Busanello J, Kerber NPC, Fernandes GFM, Zacarias CC, Cappellaro J, Silva ME. Humanização do parto e a formação dos profissionais da saúde. Ciênc Cuid Saúde. 2011;10(1):169-75.
24. Sanches NC, Mamede FV, Vivancos RBZ. [The profile of women who have experienced cesarean section and obstetric care at a public maternity hospital in Ribeirao Preto]. Texto Contexto Enferm. 2012;21(2):418-26. Portuguese

25. Haddad SEMT, Cececatti JG. [Strategies directed to professionals for reducing unnecessary cesarean sections in Brazil]. Rev Bras Ginecol Obstet. $2011 ; 33(5): 252-62$. Portuguese.

26. Nagahama EEI, Santiago SM. [Humane childbirth and type of childbirth: an evaluation of care provided by the Brazilian national health system in a city in the south of Brazil]. Rev Bras Saúde Mater Infant. $2011 ; 11(4): 415-25$. Portuguese.

27. Giglio MRP, França E, Lamounier JA. [Evaluation of the quality of care for normal delivery]. Rev Bras Ginecol Obstet. 2011;33(10)297. 303. Portuguese.

28. Rosenblatt RA. The perinatal paradox: doing more and accomplishing less. Health Aff (Millwood). 1989;8(3):158-68.

29. Diniz SG. Gênero, saúde materna e o paradoxo perinatal. Rev Bras Crescimento Desenvolv Hum. 2009;19(2):313-26. 